\title{
Formation of interfacial $\eta^{\prime}-\mathrm{Cu}_{6} \mathrm{Sn}_{5}$ in $\mathrm{Sn}-\mathbf{0 . 7 C u} / \mathrm{Cu}$ solder joints during isothermal aging - ERRATUM
}

\author{
Zhongbing Luo, Lai Wang, Qinqin Fu, Chongqian Cheng, and Jie Zhao
}

doi: 10.1557/jmr.2011.179, Published by Cambridge University Press, 27 June 2011.

In the first paragraph, this sentence was cited incorrectly. The cited work was done by Nogita et al.

Importantly, the density changes with the structural evolutions. It may be catastrophic to the intrinsically brittle IMC as reported by Nogita et al. that more cracks were observed at the interface of $\mathrm{Sn}-\mathrm{Cu} / \mathrm{Cu}$ if no Ni was added to inhibit the transformation. ${ }^{4}$

The correct citation is as follows:

Importantly, the density changes with the structural evolutions. It may be catastrophic to the intrinsically brittle IMC as reported by Nogita et al. that more cracks were observed at the interface of $\mathrm{Sn}-\mathrm{Cu} / \mathrm{Cu}$ if no $\mathrm{Ni}$ was added to inhibit the transformation. ${ }^{8}$

The publisher regrets the error.

\section{Reference}

Zhongbing Luo, Lai Wang, Qinqin Fu, Chongqian Cheng, and Jie Zhao: Formation of interfacial $\eta^{\prime}-\mathrm{Cu}_{6} \mathrm{Sn}_{5}$ in $\mathrm{Sn}-0.7 \mathrm{Cu} / \mathrm{Cu}$ solder joints during isothermal aging. J. Mater. Res. 27, 1468 (2011). 